\title{
SELETIVIDADE DE FUNGICIDAS UTILIZADOS NA CULTURA DO TOMATEIRO (Lycopersicum esculentum, MILL.) A Trichogramma pretiosum
}

\author{
CARVALHO, José Romário ${ }^{1}$ \\ PRATISSOLI, Dirceu ${ }^{2}$ \\ PAES, João Paulo Pereira ${ }^{3}$ \\ ZUIM, Vitor ${ }^{3}$ \\ STINGUEL, Priscila ${ }^{3}$ \\ MINAS , Ramon Santos de
}

SALOMÃO, Kharen Priscilla de Oliveira Silva ${ }^{4}$

\begin{abstract}
RESUMO: Este estudo avaliou, em condições laboratoriais $\left(25 \pm 1^{\circ} \mathrm{C}\right.$, UR de $70 \pm 10 \%$ e fotofase de $\left.14 \mathrm{~h}\right)$, a seletividade de 18 fungicidas utilizados na cultura do tomateiro à Trichogramma pretiosum Riley (Hymenoptera: Trichogrammatidae), que é um dos inimigos naturais mais empregados no controle biológico. Cartelas com ovos de Anagasta kuehniella (Zeller) (Lepidoptera: Pyralidae) foram tratados com os fungicidas (tratamentos) e submetidos ao parasitismo por 24 horas. Os parâmetros biológicos avaliados foram: percentual de parasitismo, percentual de emergência e razão sexual. Os fungicidas a base de CLOROTALONIL, FENAMIDONA, FLUAZINAM, HIDRÓXIDO DE COBRE e ZOXAMIDA + MANCOZEBE causaram reduções de até $80 \%$ no parasitismo de $T$. pretiosum. Os fungicidas a base de TIOFANATO METÍLICO e IPROVALICARBE + PROPINEBE foram seletivos e proporcionaram um incremento na taxa de parasitismo na ordem de 11,48 e 16,58\%, respectivamente. A emergência dos parasitoides não foi afetada significativamente apresentando valores superiores a 95\%, enquanto que a razão sexual de $T$. pretiosum sofreu uma pequena variação, 0,98 e 1,00. Diante disso, verificou-se que os fungicidas a base de TIOFANATO e IPROVALICARBE + PROPINEBE são os mais indicados para serem utilizados em associação com controle biológico, por não afetarem o parasitoide.
\end{abstract}

Palavras-chave: Insecta. Agrotóxico. Controle biológico. Parasitoide de ovos. Manejo integrado de pragas.

\section{SELECTIVITY OF FUNGICIDES USED IN TOMATO CROP (Lycopersicum esculentum, MILL.) TO Trichogramma pretiosum}

SUMMARY: This study evaluated under laboratory conditions $\left(25 \pm 1{ }^{\circ} \mathrm{C}, \mathrm{RH} 70 \pm 10 \%\right.$ and $14 \mathrm{~h}$ photophase $)$, the selectivity of 18 fungicides in tomato to Trichogramma pretiosum Riley (Hymenoptera: Trichogrammatidae), which is one of the natural enemies more employees in biocontrol. Cards with Anagasta kuehniella (Zeller) (Lepidoptera: Pyralidae) were treated with fungicides (treatments) and submitted to parasitism by 24 hours. The parameters evaluated were: percentage of parasitism, percent emergence and sex ratio. The fungicide the base CHLOROTHALONIL, FENAMIDONE, FLUAZINAM, COPPER HYDROXIDE and ZOXAMIDE + MANCOZEB caused reductions of up to $80 \%$ parasitism of $T$. pretiosum. Fungicides the base THIOPHANATE METHYL and IPROVALICARB + PROPINEB were selective and provided an increase in the rate of parasitism in order 11.48 and $16.58 \%$, respectively. The emergence of parasitoids was not significantly affected with values above $95 \%$, while the sex ratio of $T$. pretiosum suffered a slight variation, 0.98 and 1.00. Therefore, it was found that the base of fungicides THIOPHANATE and IPROVALICARB + PROPINEB are best suited for use in combination with biological control because they do not affect the parasitoid.

Keywords: Insecta. Pesticides. Biological control. Egg parasitoids. Integrated pest management.

\footnotetext{
1 Universidade Federal do Espírito Santo (UFES). Departamento de Produção Vegetal, Núcleo de Manejo Fitossanitário de Pragas e Doenças (NUDEMAFI), Mestre em Produção Vegetal - Fitossanidade.

${ }^{2}$ UFES. Departamento de Produção Vegetal, Núcleo de Manejo Fitossanitário de Pragas e Doenças (NUDEMAFI), Dr em Entomologia, Professor associado III

${ }^{3}$ UFES. Departamento de Produção Vegetal, Nucleo de Manejo Fitossanitário de Pragas e Doenças, Graduando (a) em Agronomia

${ }^{4}$ UFES. Departamento de Produção Vegetal, Nucleo de Manejo Fitossanitário de Pragas e Doenças, Mestranda (o) do Programa de Pós Graduação em Produção Vegetal.
} 


\section{INTRODUÇÃO}

O tomateiro (Lycopersicum esculentum, Mill.), cultivado em todas as regiões brasileiras, é uma cultura considerada de alto risco, em virtude da infestação por diversas pragas e doenças, tanto nas lavouras destinadas ao consumo in natura, como para indústria (SOUZA; REIS, 2003; LUZ et al., 2007). Desta maneira, para se obter uma produção viável os agricultores fazem o uso indiscriminado dos agrotóxicos (CARVALHO et al., 2003).

Concomitantemente, para alguns ingredientes ativos ainda não existem informações suficientes quanto a sua eficiência quando empregados em associação com outros métodos de controles (BASTOS et al., 2007). Tal informação é crucial para a integração desta tática com outros métodos de Manejos Fitossanitário de Pragas (MFP), incluindo o uso de produtos químicos menos tóxicos e, ou seletivos (ALVES et al., 2007). Entre as táticas de controle pode-se destacar o biológico com o uso de inimigos naturais.

Parasitoides do gênero Trichogramma têm sido amplamente utilizados em programas de controle biológico de pragas (ALMEIDA et al., 2010). Dentre as diversas espécies, destaca-se Trichogramma pretiosum Riley, 1879, (Hymenoptera: Trichogrammatidae) devido a sua ampla distribuição e agressividade, sendo relatada em 18 hospedeiros e em 13 culturas (ZUCCHI ; MONTEIRO, 1997; PRATISSOLI et al., 2004; DELPUECH et al., 2010).

No entanto, devido à utilização demasiada quantidade de agrotóxicos para o controle de insetospraga e doenças na cultura do tomateiro, o controle biológico pode sofrer alguma interferência, devido a ação destes produtos. Com isso, faz-se necessário a realização de testes, verificando a influência dos agrotóxicos sobre os agentes do controle biológico, gerando informações que possam auxiliar na tomada de decisão em programas de Manejo Fitossanitário de Pragas (PRATISSOLI et al., 2002; ZANUNCIO et al., 2003).

Testes de seletividade de agrotóxicos à adultos de diferentes espécies de Trichogramma têm sido realizados no Brasil (DEGRANDE et al., 2002). O protocolo de padronização desses testes, visando avaliar a toxicidade e o impacto de agrotóxicos sobre Trichogramma, foi desenvolvido pela International Organization for Biological Control of Noxious Plants and Animals/ West Paleartic Region Section (IOBC/ WPRS), um grupo de trabalho que padroniza testes de seletividade (HASSAN et al., 1998; PRATISSOLI et al., 2009).

O objetivo deste trabalho foi avaliar a seletividade de fungicidas recomendados para a cultura do tomateiro sobre o parasitismo, emergência (viabilidade do parasitismo) e razão sexual do parasitoide de ovos T. pretiosum em ovos do hospedeiro alternativo Anagasta kuehniella (Zeller) (Lepidoptera: Pyralidae) em condições de laboratório.

\section{MATERIAL E MÉTODOS}

O trabalho foi realizado no setor de entomologia do Núcleo de Desenvolvimento de Manejo Fitossanitário de Pragas e Doenças (NUDEMAFI) do Centro de Ciências Agrárias da Universidade Federal do Espírito Santo (CCA-UFES), em Alegre - ES.

Criação e manutenção de Trichogramma pretiosum: Na manutenção e multiplicação do parasitoide T. pretiosum, empregou-se o hospedeiro alternativo Anagasta kuehniella (Zeller) (Lepidoptera: Pyralidae), de acordo com a metodologia desenvolvida por Pratissoli et al. (2010). Para a manutenção do 
hospedeiro utilizou-se uma dieta à base de farinha de trigo integral (70\%), farinha de milho (27\%) e levedura de cerveja (3\%).

Para criação do parasitoide, ovos do hospedeiro foram aderidos às cartelas de cartolina azul $(8 \mathrm{~cm}$ x $2 \mathrm{~cm})$, utilizando-se goma arábica diluída à $50 \%(\mathrm{~m} / \mathrm{v})$. Esses ovos foram, posteriormente, submetidos à inviabilização sob lâmpada germicida por um tempo de 50 minutos, conforme descrito por Pratissoli et al. (2010), sendo posteriormente expostos ao parasitismo por um período de 24 horas e mantidos em câmara climatizada a $25 \pm 1^{\circ} \mathrm{C}$, UR de $70 \pm 10 \%$ e fotofase de 14 horas.

Bioensaio com os fungicidas: Todos os fungicidas testados estão registrados para o controle de doenças na cultura do tomate em território nacional (AGROFIT, 2011). As formulações comerciais testadas foram: AZOXISTROBINA (500 g i.a./kg, granulado dispersível, CCAB Agro, São Paulo, SP, Brasil), CLORIDRATO DE PROPAMOCARBE (720 g i.a./L, suspensão de encapsulado, Cross Link, Barueri, SP, Brasil), CLOROTALONIL + OXICLORETO DE COBRE (250 + 300 g i.a./kg, pó molhável, Iharabras, Sorocaba, SP, Brasil), CLOROTALONIL 1 (500 g i.a./L, suspensão concentrada, Syngenta, São Paulo, SP, Brasil), CLOROTALONIL 2 (750 g i.a./kg, pó molhável, Iharabras, Sorocaba, SP, Brasil), DIFENOCONAZOLE (250 g i.a./L, concentrado emulsionável, Syngenta, São Paulo, SP, Brasil), DIMETOMORFE (500 g i.a./kg, pó molhável, BASF S.A., São Paulo, Brazil), FENAMIDONA (500 g i.a./L, suspensão concentrada, Bayer CropScience, São Paulo, SP, Brasil), FLUAZINAM (500 g i.a./L, suspensão concentrada, CCAB Agro, São Paulo, SP, Brasil), HIDRÓXIDO DE COBRE (537,4 g i.a./L, suspensão concentrada, Oxiquímica Agrociência, Jaboticabal, SP, Brasil), IPROVALICARBE + PROPINEBE (55 + 613 g i.a./kg, pó molhável, Bayer CropScience, São Paulo, SP, Brasil), OXICLORETO DE COBRE (840 g i.a./kg, pó molhável, Arysta Lifescience, São Paulo, SP, Brasil), PROCIMIDONA (500 g i.a./kg, pó molhável, Nufarm, Maracanaú, CE, Brasil), TEBUCONAZOL (250 g i.a./kg, pó molhável, Bayer CropScience, São Paulo, SP, Brasil), TIOFANATO METÍLICO 1 (500 g i.a./L, suspensão concentrada, Helm, São Paulo, SP, Brasil), TIOFANATO METÍLICO 2 (700 g i.a./kg, pó molhável, Iharabras, Sorocaba, SP, Brasil), ZOXAMIDA + MANCOZEBE $(73+727$ g i.a./kg, pó molhável, Cross Link, Barueri, SP, Brasil).

Para todos os produtos empregou-se a maior concentração recomendada pelo fabricante, e no tratamento testemunha utilizou-se somente água destilada.

Cada fêmea foi individualizada em tubo Duran $(3,0 \times 0,5 \mathrm{~cm})$ e que foi fechado com filme de polietileno. Foram aderidos às cartelas de cartolina azul $(2,5 \times 0,3 \mathrm{~cm}) 40$ ovos de A. kuehniella (idade $<12$ horas) com goma arábica $(50 \% \mathrm{~m} / \mathrm{v})$, sendo posteriormente inviabilizados sob lâmpada germicida. Após a inviabilização, as cartelas contendo os ovos foram imersas nas caldas, contendo fungicidas, por cinco segundos. Em seguida as cartelas foram colocadas em bandejas contendo papel toalha para retirada do excesso de umidade, e foram ofertadas aos parasitoides que se encontravam em câmara climatizada reguladas a $25 \pm 1^{\circ} \mathrm{C}$, UR de $70 \pm 10 \%$ e fotofase de 14 horas.

Após 24 horas, as fêmeas foram descartadas e os ovos mantidos nas mesmas condições anteriores. Após a emergência avaliou-se o número de ovos parasitados por fêmea, percentual de emergência e razão sexual.

Análise dos dados obtidos: O número médio de ovos parasitados por fêmea de T. pretiosum foi utilizado para calcular o percentual de parasitismo. A redução no parasitismo para cada fungicida foi determinada através da comparação com a testemunha (água destilada) e calculada por meio da fórmula: $\mathrm{RP}=(1-\mathrm{Rt} / \mathrm{Rc})^{*} 100$, sendo RP a porcentagem de redução no parasitismo; Rt o valor do parasitismo 
médio para cada produto e Rc o parasitismo médio observado para o tratamento testemunha (negativa), conforme Hassan et al., 2000. Com base nestas porcentagens de redução no parasitismo, os fungicidas testados foram classificados segundo IOBC/WPRS em: 1) inócuo $(<30 \%) ; 2)$ levemente nocivo (30-

$79 \%)$; 3 ) moderadamente nocivo (80-99\%) e; 4) nocivo (> 99\%).

O delineamento utilizado foi o inteiramente casualizado com 18 fungicidas e uma testemunha (tratamentos) contendo 15 repetições cada. Os resultados obtidos referentes ao parasitismo, percentual de emergência e razão sexual foram submetidos à análise de variância (ANOVA) e as médias comparadas pelo teste de Scott-Knott $(\mathrm{p} \leq 0,05)$.

\section{RESULTADOS E DISCUSSÃO}

O parasitismo de $T$. pretiosum foi afetado pelos fungicidas testados, que por sua vez oscilou entre 6,7 e 45,7\%. Os fungicidas a base de CLOROTALONIL, FENAMIDONA, FLUAZINAM, HIDRÓXIDO DE COBRE e ZOXAMIDA + MANCOZEBE promoveram redução do parasitismo (Tabela 1). Independente da concentração testada, os fungicidas à base de CLOROTALONIL foram semelhantes entre as concentrações desse e ao fungicida à base de FENAMIDONA. Perante a redução de parasitismo, os fungicidas à base de CLOROTALONIL e FENAMIDONA enquadraram-se na classificação toxicológica da IOBC/WPRS como levemente nocivos. Este resultado possibilita sua utilização em associação com liberações do parasitoide T. pretiosum. Os fungicidas à base de FLUAZINAM, HIDRÓXIDO DE COBRE e ZOXAMIDA + MANCOZEBE proporcionaram maior redução no parasitismo $(6,7 ; 7,7$ e 7,8\% de parasitismo, respectivamente), enquadrando-se como moderadamente nocivos, pela classificação toxicológica da IOBC/WPRS e desta forma, não possibilitam a utilização em associação com o parasitoide.

Tabela 1. Percentual de parasitismo ( $\pm \mathrm{EP})$ por fêmea, redução no parasitismo (RP) de T. pretiosum em relação à testemunha e classes de seletividade dos fungicidas recomendados para a Cultura do Tomateiro. Temperatura $25 \pm 1{ }^{\circ} \mathrm{C}$; UR: $70 \pm 10 \%$; fotofase: $14 \mathrm{~h}$.

\begin{tabular}{|c|c|c|c|}
\hline Tratamento & Parasitismo $(\%)^{*}$ & $\mathrm{RP}(\%))^{1}$ & Classe $^{2}$ \\
\hline AZOXISTROBINA & $42,6 \pm 2,24 \mathrm{a}$ & $(8,67)$ & 1 \\
\hline CLORIDRATO DE PROPAMOCARBE & $43,1 \pm 1,88$ a & $(9,95)$ & 1 \\
\hline $\begin{array}{c}\text { CLOROTALONIL + OXICLORETO DE } \\
\text { COBRE }\end{array}$ & $21,5 \pm 4,18 b$ & 45,15 & 2 \\
\hline CLOROTALONIL 1 & $26,8 \pm 4,91 \mathrm{~b}$ & 31,63 & 2 \\
\hline CLOROTALONIL 2 & $22,1 \pm 4,76 b$ & 43,62 & 2 \\
\hline DIFENOCONAZOL & $39,8 \pm 1,8 \mathrm{a}$ & $(1,53)$ & 1 \\
\hline DIMETOMORFE & $35,5 \pm 3,86 \mathrm{a}$ & 9,44 & 1 \\
\hline FAMOXADONA & $41,5 \pm 1,72 \mathrm{a}$ & $(5,87)$ & 1 \\
\hline FENAMIDONA & $21,7 \pm 4,37 \mathrm{~b}$ & 44,64 & 2 \\
\hline FLUAZINAM & $6,7 \pm 3,61 \mathrm{c}$ & 82,91 & 3 \\
\hline HIDRÓXIDO DE COBRE & $7,7 \pm 3,54 \mathrm{c}$ & 80,36 & 3 \\
\hline IPROVALICARBE + PROPINEBE & $45,7 \pm 1,58 \mathrm{a}$ & $(16,58)$ & 1 \\
\hline OXICLORETO DE COBRE & $40,7 \pm 1,81 \mathrm{a}$ & $(3,83)$ & 1 \\
\hline PROCIMIDONA & $41,7 \pm 2,74 \mathrm{a}$ & $(6,38)$ & 1 \\
\hline TEBUCONAZOL & $40,0 \pm 1,46 \mathrm{a}$ & $(2,04)$ & 1 \\
\hline TIOFANATO METÍLICO 1 & $43,7 \pm 2,13 \mathrm{a}$ & $(11,48)$ & 1 \\
\hline TIOFANATO METÍLICO 2 & $30,7 \pm 2,73 \mathrm{a}$ & 21,68 & 1 \\
\hline ZOXAMIDA + MANCOZEBE & $7,8 \pm 3,87 \mathrm{c}$ & 80,10 & 3 \\
\hline Testemunha & $39,2 \pm 2,23 \mathrm{a}$ & ----- & ------ \\
\hline $\mathrm{F}$ & 17,964 & & \\
\hline GLR & 266 & & \\
\hline $\mathrm{P}$ & $<0,001$ & & \\
\hline
\end{tabular}

Médias seguidas de mesma letra não diferem estatisticamente entre si pelo Teste de Scott-Knott a 5\% de probabilidade; 
${ }^{T} \mathrm{RP}=$ Redução no parasitismo comparado com a testemunha. Valor entre parênteses caracteriza aumento no parasitismo;

${ }^{2}$ Classes da IOBC/WPRS para teste de seletividade sobre Trichogramma: $1=$ inócuo $(<30 \%), 2=$ levemente nocivo (30-79\%), 3 = moderadamente nocivo ( $80-99 \%), 4$ = nocivo (> 99\%).

Os demais fungicidas não diferiram do tratamento testemunha, enquadrando-se na classe inócuos da IOBC/WPRS, sendo desta maneira, os melhores para utilização em associação com este inimigo natural. Todavia, dentre os fungicidas classificados como inócuos, somente o DIMETOMORFE e TIOFANATO METÍLICO 2 causaram redução no parasitismo (Tabela 1). Para os demais fungicidas ocorreu incremento da taxa de parasitismo, que oscilou de 2,04\%, para TEBUCONAZOL, a 16,58\%, para IPROVALICARBE + PROPINEBE.

A ação negativa de CLOROTALONIL e MANCOZEBE foi reportada por Pratissoli et al. (2010) para Trichogramma atopovirilia Oatman e Platner. Segundo esses autores esses produtos causaram uma redução de 43,37 e 7,38\%, respectivamente, no parasitismo de ovos de Diaphania hyalinata Linnaeus (Lepidoptera: Pyralidae). Manzoni et al. (2006) reportaram a redução do parasitismo de T. pretiosum em ovos de A. kuehniella causada por MANCOZEBE (47,79\%). No presente estudo, a associação entre ZOXAMIDA e MANCOZEBE intensificou a toxicidade aos adultos de $T$. pretiosum, reduzindo bruscamente o parasitismo $(80,10 \%)$.

Resultado contrastante foi relatado por Giolo et al. (2007) para TEBUCONAZOL. Segundo esses autores o TEBUCONAZOL 200 EC proporcionou uma redução de 42,7\% no parasitismo de $T$. pretiosum em ovos de A. kuehniella enquadrando-o na classe 2 da IOBC/WPRS, enquanto que no presente estudo ocorreu um incremento no parasitismo (2,04\%), o que o classificou como inócuo. Pasini et al. (2007), por sua vez, reportaram uma redução do parasitismo de T. pretiosum em ovos de A. kuehniella na ordem de $100 \%$ quando tratados com TEBUCONAZOL 200 EC, o que o classificou como nocivo (classe 4 da IOBC/WPRS). Isso, em hipótese, pode estar diretamente associado à composição da formulação utilizada, visto que no presente estudo utilizou-se a formulação WP (pó molhável), bem como estar diretamente relacionado com a metodologia empregada. Além disso, por se tratarem de populações diferentes de $T$. pretiosum, essas podem ter sido coletadas em localidades cujo nível de exposição a esse produto seja diferente, gerando desta forma, diferentes níveis de tolerância ao princípio ativo.

$\mathrm{O}$ incremento no desempenho de $T$. pretiosum pode estar diretamente aliado, em hipótese, à ocorrência de hormese, fato este reportado por Pratissoli et al. (2010) e, que pode ser visualizado nos resultados do presente estudo. A hormese baseia-se no fornecimento de respostas biológicas favoráveis a organismos benéficos, quando expostos aos agentes estressores em quantidades pequenas, como efeitos estimulatórios (CALABRESE, 2010; GUEDES; MAGALHÃES; COSME, 2009; MATTSON ; CALABRESE, 2010). Contudo, a confirmação deste evento carece de estudos minuciosos acerca da análise química para os organismos biológicos com maior complexidade, tais como endoparasitoides.

$O$ percentual de emergência não apresentou diferença significativa entre os diferentes tratamentos (Tabela 2). Em todos os tratamentos a emergência foi superior a $99 \%$, exceto para os fungicidas FENAMIDONA, FLUAZINAM e HIDRÓXIDO DE COBRE (94,4; 97,4 e 95,8\%, respectivamente).

Tabela 2. Percentual de emergência e razão sexual $( \pm \mathrm{EP})$ de descendentes de $T$. pretiosum em ovos de $A$. kuehniella tratados com fungicidas recomendados para a Cultura do Tomateiro. Temperatura $25 \pm 1{ }^{\circ} \mathrm{C}$; UR: $70 \pm 10 \%$; fotofase: $14 \mathrm{~h}$.

\begin{tabular}{ccc}
\hline Tratamento & ${\text { Emergência }(\%)^{\mathrm{ns}}}$ & Razão sexual $^{* *}$ \\
\hline AZOXISTROBINA & $99,6 \pm 0,39$ & $0,98 \pm 0,01 \mathrm{~b}$ \\
CLORIDRATO DE PROPAMOCARBE & $99,6 \pm 0,35$ & $0,99 \pm 0,00 \mathrm{a}$
\end{tabular}


CLOROTALONIL + OXICLORETO DE

COBRE

CLOROTALONIL 1

CLOROTALONIL 2

DIFENOCONAZOL

DIMETOMORFE
$96,9 \pm 1,24$

$99,5 \pm 0,47$

$97,2 \pm 1,55$

$100,0 \pm 0,00$

$99,4 \pm 0,55$
$1,00 \pm 0,00 \mathrm{a}$

$1,00 \pm 0,00 \mathrm{a}$

$1,00 \pm 0,00$ a

$0,99 \pm 0,00$ a

$1,00 \pm 0,00$ a 
Tabela 2. Percentual de emergência e razão sexual $( \pm \mathrm{EP})$ de descendentes de $T$. pretiosum em ovos de $A$. kuehniella tratados com fungicidas recomendados para a Cultura do Tomateiro. Temperatura $25 \pm 1^{\circ} \mathrm{C}$; UR: $70 \pm 10 \%$; fotofase: $14 \mathrm{~h}$.

\begin{tabular}{|c|c|c|}
\hline FAMOXADONA & $100,0 \pm 0,00$ & $0,99 \pm 0,00 \mathrm{a}$ \\
\hline FENAMIDONA & $94,4 \pm 1,34$ & $1,00 \pm 0,00 \mathrm{a}$ \\
\hline FLUAZINAM & $97,4 \pm 2,56$ & $1,00 \pm 0,00 \mathrm{a}$ \\
\hline HIDRÓXIDO DE COBRE & $95,8 \pm 4,16$ & $1,00 \pm 0,00 \mathrm{a}$ \\
\hline IPROVALICARBE + PROPINEBE & $99,7 \pm 0,28$ & $1,00 \pm 0,00 \mathrm{a}$ \\
\hline OXICLORETO DE COBRE & $100,0 \pm 0,00$ & $1,00 \pm 0,00 \mathrm{a}$ \\
\hline PROCIMIDONA & $99,5 \pm 0,49$ & $0,99 \pm 0,00 \mathrm{a}$ \\
\hline TEBUCONAZOL & $99,6 \pm 0,33$ & $1,00 \pm 0,00 \mathrm{a}$ \\
\hline TIOFANATO METÍLICO 1 & $99,6 \pm 0,39$ & $0,99 \pm 0,00 \mathrm{a}$ \\
\hline TIOFANATO METÍLICO 2 & $100,0 \pm 0,00$ & $1,00 \pm 0,00 \mathrm{a}$ \\
\hline ZOXAMIDA + MANCOZEBE & $100,0 \pm 0,00$ & $1,00 \pm 0,00 \mathrm{a}$ \\
\hline Testemunha & $100,0 \pm 0,00$ & $1,00 \pm 0,00 \mathrm{a}$ \\
\hline $\mathrm{F}$ & 1,697 & 6,778 \\
\hline GLR & 266 & 266 \\
\hline $\mathrm{P}$ & 0,051 & $<0,001$ \\
\hline
\end{tabular}

\footnotetext{
${ }^{\mathrm{T}}$ Médias seguidas de mesma letra diferem estatisticamente pelo teste de Scott-Knott a 5\% de probabilidade;

${ }^{\text {ns }}$ Diferença não significativa pela ANOVA (teste de $F$ ) a $5 \%$ de probabilidade.
}

Pratissoli et al. (2010) relataram que os fungicidas CLOROTALONIL 500 SC, TIOFANATO METÍLICO 700 WP e TEBUCONAZOL 250 WP reduziram o percentual de emergência de descendentes de $T$. atopovirilia em ovos de D. hyalinata na ordem de 73,77; 75,62 e 79,35\%, respectivamente. Este comportamento pode, em hipótese, estar diretamente associado a características intrínsecas do hospedeiro, como espessura e composição físico-química do cório do ovo.

Nesse contexto, a ação atóxica de algumas moléculas pode ocorrer devido à absorção dessas nos tecidos gordurosos, bem como ser devido a metabolização ou excreção dessas moléculas pelos insetos (FOESTER, 2002).

Para os resultados obtidos com a emergência dos machos e fêmeas, verificou-se que houve alteração na razão sexual para os tratamentos com fungicidas (Tabela 2). Somente o tratamento com o fungicida AZOXISTROBINA diferiu dos demais e da testemunha. No entanto, para todos os tratamentos a razão sexual foi superior a 0,98 , ou seja, $98 \%$ de fêmeas, corroborando com os resultados de Carvalho et al. (2003). A razão sexual de Trichogramma sp. pode sofrer efeito de vários fatores, entre esses a manutenção de sucessivas gerações em laboratório (STEIN ; PARRA, 1987), o tamanho do hospedeiro (BAI et al., 1992), e o efeito de temperatura e antibióticos (STOUTHAMER, 1990).

\section{CONCLUSÃO}

1. O parasitismo de T. pretiosum foi afetado pelos tratamentos com fungicida;

2. A razão sexual foi afetada somente pelo fungicida AZOXISTROBINA;

3. Os fungicidas a base de CLOROTALONIL, FENAMIDONA, FLUAZINAM, HIDRÓXIDO DE COBRE e ZOXAMIDA + MANCOZEBE são indicados para uso em associação com T. pretiosum;

4. Os fungicidas a base de TIOFANATO METÍLICO e IPROVALICARBE + PROPINEBE, além de seletivos, promovem incremento significativo no parasitismo de T. pretiosum.

\section{AGRADECIMENTOS}

Às instituições de fomento FINEP, CNPq e FAPES pelo financiamento. 


\section{REFERÊNCIAS}

ALMEIDA, G. D.et al. Effect of azadirachtin on the control of Anticarsia gemmatalisand its impact on Trichogramma pretiosum. Phytoparasitica, v. 38, p. 413-419, 2010.

ALVES, F.R.et al. Manejo fitossanitário de doenças e pragas: novas perspectivas. In: JESUS JUNIOR, W.C. et al. (Eds.). Atualidades em defesa fitossanitária. Alegre, Universidade de Federal do Espírito Santo, 2007. cap. 13, p. 383-416.

ANÔNIMO, 2011. AGROFIT.: Sistema de Agrotóxicos Fitossanitários. Ministério da Agricultura, Pecuária e Abastecimento, Coordenação Geral de Agrotóxicos e Afins/DFIA/DAS, Brasília, Brazil. Disponível em: http://agrofit.agricultura.gov.br/agrofit_cons/principal_agrofit_cons Acessado em 25 jun. 2011.

BAI, B.et al. The effect of host size on quality attributes of the egg parasitoid, Trichogramma pretiosum. Entomology Experimental Aplied, v. 64, n. 1, p. 37-48, 1992.

BASTOS C.S.; ALMEIDA R.P.; SUINAGA F.A. Selectivity of pesticides used on cotton (Gossypium hirsutum) to Trichogramma pretiosum reared on two laboratory-reared hosts. Pest Management Science, v. 62 , p. 92-98, 2007.

CALABRESE, E. J. Hormesis: once marginalized, evidence now supports hormesis as the most fundamental dose response. In: MATTSON, M. P.; CALABRESE, E. J. (Eds.). Hormesis: a revolution in biology, toxicology and medicine. New York: Springer, cap. 2, p. 1-14, 2010.

CARVALHO, G. A.; PARRA, J. R. P.; BAPTISTA, G. C. Bioatividade de produtos fitossanitários utilizados na cultura do tomateiro (Lycopersicon esculentum MILL.) a Trichogramma pretiosum Riley, 1879 (Hymenoptera: Trichogrammatidae) nas gerações F1 e F2. Ciência e Agrotecnologia, v. 27, n. 2, p. 261-270, 2003

DEGRANDE, P.E. et al. Metodologia para avaliar o impacto de pesticidas sobre inimigos naturais. In: PARRA, J.R.P.et al. (Eds.). Controle biológico no Brasil: parasitoides e predadores. São Paulo: Manole, 2002. cap.5, p.71-93.

DELPUECH, J. M.; DUPONT, C.; ALLEMAND, R. Decrease in fecundity induced by interspecific mating between two Trichogramma parasitoid species. Journal Economic Entomology, v. 103, n. 2, p. 308-313, 2010.

FOERSTER, L. A. Seletividade de inseticidas a predadores e parasitoides. In: PARRA, J.R.P., P.S.M. BOTELHO, B.S. CORRÊA-FERREIRA, J.M.S. Bento (Eds.) Controle biológico no Brasil: parasitoides e predadores. São Paulo: Manole, p. 95-114, 2002.

GIOLO, F. P.et al. Toxicidade de produtos fitossanitários utilizados na cultura do pessegueiro sobre adultos de Trichogramma pretiosum. Bragantia, v. 66, p.423-431, 2007.

GUEDES, R. N. C.; MAGALHÃES, L. C.; COSME, L. V. Stimulatory sublethal response of a generalist predator to permethrin: hormesis, hormoligosis, or homeostatic regulation? Journal Economy Entomology, v. 102, p. 170-176, 2009.

HASSAN, S.A.et al. The side effects of pesticides on the egg parasitoid Trichogramma caecoeciae Marchal (Hym., Trichogrammatidae), acute dose-response and persistence tests. Journal of Applied Entomology, v. 122, n. 9-10, p. 569-573, 1998.

HASSAN, S.A.et al. A laboratory method to evaluate the side effects of plant protection products on Trichogramma cacoeciae Marchal (Hym., Trichogrammatidae). In: CANDOLFI, M.P. et al. (Eds.). Guidelines to evaluate side-effects of plant protection products to non-target arthropods. IOBC/ WPRS, Gent. p. 107-119, 2000. 
LUZ, J. M .Q.; SHINZATO, A. V.; SILVA, M. A. D. Comparação dos sistemas de produção de tomate convencional e orgânico em cultivo protegido. Bioscience Journal, v. 23, p. 7-15, 2007.

MANZONI, C. G. et al. Susceptibilidade de Adultos de Trichogramma pretiosum Riley (Hymenoptera: Trichogrammatidae) a Fungicidas Utilizados no Controle de Doenças da Macieira. Neotropical Entomology, v. 35, n. 2, p. 223-230, 2006.

MATTSON, M. P.; CALABRESE, E. J. Hormesis: What It Is and Why It Matters? In: MATTSON, M. P.; CALABRESE, E. J. (Eds.). Hormesis: a revolution in biology, toxicology and medicine. New York: Springer, cap. 1, p. 1-14, 2010.

PASINI, R. A.et al. Redução da capacidade de parasitismo de Trichogramma pretiosum RILEY, 1879 (HYMENOPTERA: TRICHOGRAMMATIDAE) quando exposto a inseticidas e fungicidas utilizados na cultura do milho. In: __ CONGRESSO DE INICIAÇÃO CIENTÍFICA, 14 e ENCONTRO DE PÓS GRADUAÇÃO, 9. Anais... 2007.

PRATISSOLI, D.et al. Características biológicas de Trichogramma exiguum em ovos de Anagasta kuehniella e Sitotroga cerealella. Idésia, v. 28, n. 1, p. 39-42, 2010.

PRATISSOLI, D.et al. Ocorrência de Trichogramma pretiosum em áreas comerciais de tomate, no Espirito Santo, em regiões de diferentes altitudes. Horticultura Brasileira, Brasília, v. 21, n. 1, p. 73-76, 2002.

PRATISSOLI, D.et al. Efeito da ausência de hospedeiro e de alimento sobre aspectos biológicos de Trichogramma pretiosum Riley (Hymenoptera: Trichogrammatidae). Acta Scientiarum Agronomy, v. 26, p. 281-286, 2004.

PRATISSOLI, D.et al. Side effects of fungicides used in cucurbitaceous crop on Trichogramma atopovirilia OATMAN ; PLATNER (HYMENOPTERA: TRICHOGRAMATIDAE). Chilean Journal Of Agricultural Research, v. 70, n. 2, p. 323-327, 2010.

PRATISSOLI, D. et al. Seletividade de inseticidas a Trichogramma pretiosum Riley (Hymenoptera: Trichogrammatidae) em diferentes hospedeiros. Boletín de Sanidad Vegetal Plagas, v. 35, n. 3, p. 347 $353,2009$.

SOUZA, J.C.; REIS, P.R. Principais pragas do tomate para mesa: bioecologia, dano e controle. Informe Agropecuário, v.24, p.79-92, 2003.

STEIN, C.P.; PARRA, J.R.P. Uso da radiação ultravioleta para inviabilizar ovos de Anagasta kuehniella (Zeller, 1879) visando estudos com Trichogramma spp. Anais da Sociedade Entomológica do Brasil, v. 16, p. 229-231, 1987.

STOUTHAMER, R.; LUCK, R. F.; HAMILTON, W. D. Antibiotics cause parathenogenetic Trichogramma (Hymenoptera: Trichogrammatidae) to revert to sex. Proceedings of Natural Academic Science, v. 87, p. 2424-2447, 1990.

VIANNA, U.R.et al. Insecticide toxicity to Trichogramma pretiosum (Hymenoptera: Trichogrammatidae) females and effect on descendant generation. Ecotoxicology, v. 18, p. 180-186, 2009.

ZANUNCIO, T.V.et al. Permethrin-induced hormesis on the predator Supputius cincticeps (Stål, 1860) (Heteroptera: Pentatomidae). Crop Protetion, v. 22, p. 941-947, 2003.

ZUCCHI, R. A.; MONTEIRO, R. C. O gênero Trichogramma na América do Sul. In: PARRA, J. R. P.; ZUCCHI, R. A. (Eds.). Trichogramma e o controle biológico aplicado. Piracicaba: FEALQ, cap.2, p.41-66, 1997. 